\title{
Application of Koszul complex to Wronski relations for $U\left(\mathfrak{g l}_{n}\right)$
}

Tôru Umeda

\begin{abstract}
Explicit relations between two families of central elements in the universal enveloping algebra $U\left(\mathfrak{g l}_{n}\right)$ of the general linear Lie algebra $\mathfrak{g l}_{n}$ are presented. The two families of central elements in question are the ones expressed respectively by the determinants and the permanents: the former are known as the Capelli elements, and the latter are the central elements obtained by Nazarov. The proofs given are based on the exactness of the Koszul complex and the EulerPoincaré principle.
\end{abstract}

Mathematics Subject Classification (2000). 17B35, 15A15, 16Exx.

Keywords. Center of universal enveloping algebra, Capelli elements, Koszul complex, Wronski relations.

\section{Introduction}

For the universal enveloping algebra $U\left(\mathfrak{g l}_{n}\right)$ of the general linear Lie algebra $\mathfrak{g l}_{n}$, several explicit families of central elements are known. In the present paper we treat the relations between the two well-known families: one expressed in terms of determinants, known as the Capelli elements, and the other in terms of permanents (see, e.g., [Ca2], [HU], [Na1], [MN] and [U4]). Explicit forms of these central elements are given as follows:

$$
\begin{aligned}
C_{k}(u) & =\frac{1}{k !} \sum_{\sigma \in \mathfrak{S}_{k}} \sum_{1 \leq i_{1}, \cdots, i_{k} \leq n} \operatorname{sgn}(\sigma) \prod_{s}\left(E_{i_{\sigma(s)} i_{s}}+(k-s-u) \cdot \delta_{i_{\sigma(s)} i_{s}}\right), \\
D_{k}(u) & =\frac{1}{k !} \sum_{\sigma \in \mathfrak{S}_{k}} \sum_{1 \leq i_{1}, \cdots, i_{k} \leq n} \prod_{s}\left(E_{i_{\sigma(s)} i_{s}}-(k-s-u) \cdot \delta_{i_{\sigma(s)} i_{s}}\right) .
\end{aligned}
$$

Here $u$ is a parameter and $\left\{E_{i j}\right\}$ are the standard basis elements of $\mathfrak{g l}_{n}$ given by the matrix units. The factors in the product with index $s=1,2, \cdots, k$ are arranged from left to right.

The $C_{k}$ and $D_{k}$ and their relations may also be described using Yangians (see Theorem 3 in [Na1], and also [MN], [M2], [OO]). Classically such relations have 
well-understood counterparts in the theory of symmetric polynomials. In this connection, $C_{k}=C_{k}(0)$ and $D_{k}=D_{k}(0)$ correspond respectively to the elementary symmetric polynomials and the complete homogeneous polynomials, so that their generating functions are essentially reciprocal. The explicit relations for the coefficients of the generating functions are sometimes attributed to Wronski (see p. 114 of $[\mathrm{A}]$ and p. 71 of [T1]). We thus call the analogous relations between the central elements $C_{k}(u)$ and $D_{k}(u)$ the Wronski relations for $U\left(\mathfrak{g l}_{n}\right)$.

Another interesting aspect of the Wronski relations is seen in a cohomological interpretation via the Koszul complex. They may be deduced from the exactness of the Koszul complex and the Euler-Poincaré principle (cf. $\S 9.3$ in [B]). In this paper, we use these ideas to find the explicit relations between the $C_{k}(u)$ and $D_{k}(u)$.

The main results in the present paper are the following:

Wronski Relations (Theorem 4.3). For $N \geq 1$, we have

$$
\begin{aligned}
& \sum_{k=0}^{N}(-)^{k} D_{k}(u) C_{N-k}(N-1-u)=0 \\
& \sum_{l=0}^{N}(-)^{l} C_{l}(l-1-v) D_{N-l}(v-l)=0 .
\end{aligned}
$$

Wronski Formulas (Theorem 4.4). The central elements $D_{k}(u)$ are $C_{k}(u)$ are respectively written in terms of each other by means of the following determinantal formulas:

$$
\begin{aligned}
D_{k}(u)=\operatorname{det}\left[\begin{array}{ccccc}
C_{1}(-u) & C_{2}(1-u) & \cdots & C_{k-1}(k-2-u) & C_{k}(k-1-u) \\
1 & C_{1}(1-u) & \cdots & C_{k-2}(k-2-u) & C_{k-1}(k-1-u) \\
& 1 & \ddots & \vdots & \vdots \\
& & \ddots & C_{1}(k-2-u) & C_{2}(k-1-u) \\
0 & & & 1 & C_{1}(k-1-u)
\end{array}\right], \\
C_{k}(u)=\operatorname{det}\left[\begin{array}{ccccc}
D_{1}(-u) & D_{2}(1-u) & \cdots & D_{k-1}(k-2-u) & D_{k}(k-1-u) \\
1 & D_{1}(1-u) & \cdots & D_{k-2}(k-2-u) & D_{k-1}(k-1-u) \\
& 1 & \ddots & \vdots & \vdots \\
0 & & \ddots & D_{1}(k-2-u) & D_{2}(k-1-u) \\
& & & 1 & D_{1}(k-1-u)
\end{array}\right] .
\end{aligned}
$$


Acknowledgements. The author expresses his sincere thanks to his friend Roger Howe for the advice to make this paper clear.

\section{Generalities on the trace}

In this section, to make our discussions clear, we gather general properties on the trace with values in non-commutative algebra. The statements are quite obvious, so that we omit their proofs.

Throughout this paper, we will work over a fixed ground field $\mathbb{K}$ of characteristic 0 . Let $V$ be a $\mathbb{K}$-vector space and $\mathcal{A}$ a $\mathbb{K}$-algebra. We consider linear maps $\Phi$ from $V$ to its coefficient extension $V \otimes \mathcal{A}=V \otimes_{\mathbb{K}} \mathcal{A}$ :

$$
\Phi: V \longrightarrow V \otimes \mathcal{A} \text {. }
$$

Our object in this section is the $\mathcal{A}$-valued trace $\operatorname{Tr}(\Phi)$ of linear maps of this type. When $V$ is finite-dimensional, the trace $\operatorname{Tr}(\Phi)$ is defined in the usual manner that

$$
\operatorname{Tr}(\Phi)=\sum_{j=1}^{n} \Phi_{j j}
$$

for $\Phi\left(v_{j}\right)=\sum_{i=1}^{n} v_{i} \otimes \Phi_{i j}$, where $\left\{v_{i}\right\}_{i=1}^{n}$ is a basis of $V$, and it is obvious that the definition does not depend on the choice of basis. Hereafter when we consider the traces, we always assume that the base vector spaces $V$ are finite-dimensional.

Lemma 1.1. Assume that the following diagram commutes:

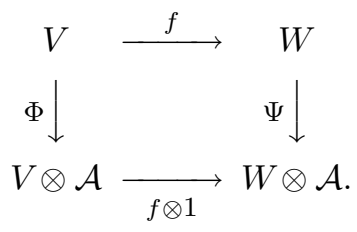

Then we see

(1) $\Phi(\operatorname{Ker} f) \subset \operatorname{Ker} f \otimes \mathcal{A}$,

(2) $\Psi(\operatorname{Im} f) \subset \operatorname{Im} f \otimes \mathcal{A}$,

(3) $\operatorname{Tr}(\Phi)=\operatorname{Tr}\left(\left.\Phi\right|_{\text {Ker } f}\right)+\operatorname{Tr}\left(\left.\Psi\right|_{\operatorname{Im} f}\right)$.

Corollary 1.2 (Euler-Poincaré Principle). Assume that $d^{i} d^{i-1}=0$ holds for all $i$ in the first row of the following commutative diagram:

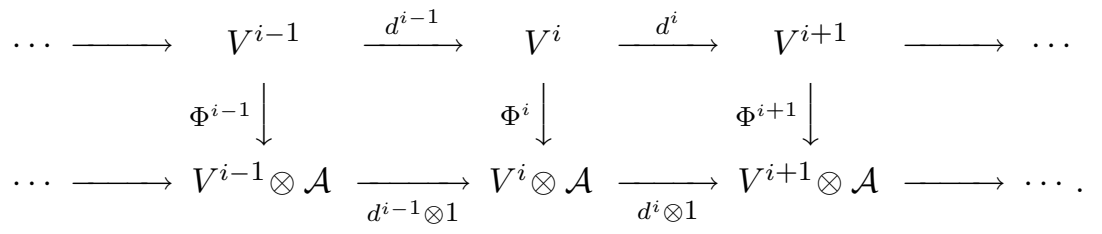


Then $\Phi^{i}$ induces a linear map $H^{i}(\Phi): H^{i}(V) \rightarrow H^{i}(V \otimes \mathcal{A})=H^{i}(V) \otimes \mathcal{A}$ of the cohomologies, and the following equality holds if the complex is finite

$$
\sum_{i}(-)^{i} \operatorname{Tr}\left(\Phi^{i}\right)=\sum_{i}(-)^{i} \operatorname{Tr}\left(H^{i}(\Phi)\right) .
$$

For two linear maps $\Phi: V \rightarrow V \otimes \mathcal{A}$ and $\Psi: W \rightarrow W \otimes \mathcal{A}$, we define their product

$$
\Phi \times \Psi: V \otimes W \longrightarrow V \otimes W \otimes \mathcal{A}
$$

via the multiplication of $\mathcal{A}$. To be more explicit, let $\left(\Phi_{p i}\right)$ and $\left(\Psi_{q j}\right)$ be the matrix expressions of $\Phi$ and $\Psi$ with respect to bases $\left\{v_{i}\right\}$ and $\left\{w_{j}\right\}$ of $V$ and of $W$. Then we have

$$
(\Phi \times \Psi)\left(v_{i} \otimes w_{j}\right)=\sum_{p, q} v_{p} \otimes w_{q} \otimes \Phi_{p i} \Psi_{q j} .
$$

We can also flip the multiplication through the isomorphism $` V \otimes W \simeq W \otimes V$, and define $\Psi \dot{\times} \Phi=`(\Phi \times \Psi)^{\check{r}}$. The explicit expression for this flipped product is given by

$$
(\Psi \check{\times} \Phi)\left(v_{i} \otimes w_{j}\right)=\sum_{p, q} v_{p} \otimes w_{q} \otimes \Psi_{q j} \Phi_{p i} .
$$

The associative laws for these products are seen as

$$
\begin{aligned}
& \left(\Phi_{1} \times \Phi_{2}\right) \times \Phi_{3}=\Phi_{1} \times\left(\Phi_{2} \times \Phi_{3}\right), \\
& \left(\Phi_{3} \check{\times} \Phi_{2}\right) \check{\times} \Phi_{1}=\Phi_{3} \check{\times}\left(\Phi_{2} \check{\times} \Phi_{1}\right) .
\end{aligned}
$$

More generally, we may consider similar products composed with the permutations of the base vector spaces when the the number of the factors is more than two. But we will not go into further details for such generalizations here.

For the trace of the product, the following are easily seen from the expressions (1.1) and (1.2).

Lemma 1.3. For linear maps $\Phi: V \rightarrow V \otimes \mathcal{A}$ and $\Psi: W \rightarrow W \otimes \mathcal{A}$, the traces of their products $\times$ and $\check{x}$ are given by $\operatorname{Tr}(\Phi \times \Psi)=\operatorname{Tr}(\Phi) \operatorname{Tr}(\Psi)$ and $\operatorname{Tr}(\Psi \check{\times} \Phi)=\operatorname{Tr}(\Psi) \operatorname{Tr}(\Phi)$.

\section{Koszul complex}

In this section, we review first basic facts on the usual Koszul (or polynomial coefficient de Rham) complex (cf. [H1] and [H2]) to fix the notation for the coefficient extension. 
Let $\mathcal{P}_{n}=\mathbb{K}\left[x_{1}, x_{2}, \cdots, x_{n}\right]$ be the polynomial ring in $n$ variables and $\Lambda_{n}$ the exterior algebra generated by the $n$ elements $e_{1}, e_{2}, \cdots, e_{n}$ subject to the relations $e_{i} e_{j}+e_{j} e_{i}=0$. We consider the polynomial coefficient exterior algebra $\Omega_{n}=$ $\mathcal{P}_{n} \otimes \Lambda_{n}$ endowed with the usual algebra structure, where the two subalgebras $\mathcal{P}_{n}$ and $\Lambda_{n}$ commute. Both $\mathcal{P}_{n}$ and $\Lambda_{n}$ have the natural graded structures, and we denote their homogeneous pieces of degrees $k$ and $l$ by $\mathcal{P}_{n}^{k}$ and $\Lambda_{n}^{l}$ respectively. The degree operators on these gradation are written as $\operatorname{deg}_{\mathcal{P}}$ and $\operatorname{deg}_{\Lambda}$. We write $\Omega_{n}^{k, l}=\mathcal{P}_{n}^{k} \otimes \Lambda_{n}^{l}$, and we sometimes abbreviate to write them as $\Omega^{k, l}=\Omega_{n}^{k, l}$.

We denote the exterior derivative by $d$ and its dual operation by $d^{*}$ :

$$
\left.d=\sum_{i=1}^{n} e_{i} \partial_{i}, \quad d^{*}=\sum_{i=1}^{n} x_{i}\right\rfloor_{i}
$$

Here $e_{i}$ and $x_{i}$ are the multiplication operators in $\Omega_{n}$, and $\partial_{i}$ and $\rfloor_{i}$ are respectively the operators of partial derivation and inner derivation. Among them the canonical commutation and anti-commutation relations hold:

$$
\left.\left.\partial_{i} x_{j}-x_{j} \partial_{i}=\delta_{i j}, \quad\right\rfloor_{i} e_{j}+e_{j}\right\rfloor_{i}=\delta_{i j} .
$$

Here the symbol $\delta_{i j}$ is Kronecker's delta. From these relations, we see $d^{2}=$ $0, d^{* 2}=0$ and

$$
d d^{*}+d^{*} d=\operatorname{deg}_{\mathcal{P}}+\operatorname{deg}_{\Lambda} .
$$

Let us consider the following two complexes:

$$
K_{N}: \quad 0 \longrightarrow \Omega^{N, 0} \stackrel{d}{\longrightarrow} \Omega^{N-1,1} \stackrel{d}{\longrightarrow} \cdots \stackrel{d}{\longrightarrow} \Omega^{0, N} \longrightarrow 0,
$$

and

$$
K_{N}^{*}: \quad 0 \longleftarrow \Omega^{N, 0} \stackrel{d^{*}}{\longleftarrow} \Omega^{N-1,1} \stackrel{d^{*}}{\longleftarrow} \cdots \stackrel{d^{*}}{\longleftarrow} \Omega^{0, N} \longleftarrow 0 .
$$

From (2.1), these complexes are exact except for $N=0$.

For an associative $\mathbb{K}$-algebra $\mathcal{A}$, we make the coefficient extension $\Omega \otimes \mathcal{A}$ of $\Omega$. Then with the differentials $d \otimes 1, d^{*} \otimes 1$ acting trivially on $\mathcal{A}$, we can regard $\Omega \otimes \mathcal{A}$ as complexes. For simplicity, we use the notation $d, d^{*}$ for these $d \otimes 1, d^{*} \otimes 1$. Also the gradings and the degree operators $\operatorname{deg}_{\mathcal{P}}$ and $\operatorname{deg}_{\Lambda}$ in $\Omega$ can be naturally extended to $\Omega \otimes \mathcal{A}$.

For $\varphi, \psi \in \Omega_{n} \otimes \mathcal{A}$, the following derivation rules are seen from the definitions:

$$
d(\varphi \psi)=d \varphi \cdot \psi+\varphi \cdot d \psi, \quad d^{*}(\varphi \psi)=d^{*} \varphi \cdot \psi+(-)^{\operatorname{deg}_{\Lambda}(\varphi)} \varphi \cdot d^{*} \psi .
$$

The exactness of the Koszul complex and Corollary 1.2 imply the following general result:

Lemma 2. If a set of linear maps

$$
\Phi^{k, l}: \Omega^{k, l} \longrightarrow \Omega^{k, l} \otimes \mathcal{A}
$$


commutes with the differential $d\left(\right.$ or $\left.d^{*}\right)$, then for $N \geq 1$ we have

$$
\sum_{k=0}^{N}(-)^{k} \operatorname{Tr}\left(\Phi^{k, N-k}\right)=0 .
$$

In the next section, for the case of the universal enveloping algebra $\mathcal{A}=U\left(\mathfrak{g l}_{n}\right)$, we will construct explicitly such families of linear maps commuting with the differential operators $d$ and $d^{*}$.

\section{Linear maps with coefficients in $U\left(\mathfrak{g l}_{n}\right)$}

In this section, we work in the algebra $\Omega_{n} \otimes U\left(\mathfrak{g l}_{n}\right)$, in which the algebra structure is defined so that two subalgebras $\Omega_{n}$ and $U\left(\mathfrak{g l}_{n}\right)$ commute.

Let us introduce some notation. For the element $E_{i j}$ of $\mathfrak{g l}_{n}$ corresponding to the matrix unit, we put

$$
E_{i j}(u)=E_{i j}+u \delta_{i j}
$$

with a parameter $u$. Define the two types of elements by

$$
\begin{aligned}
& \eta_{i}(u)=\sum_{p=1}^{n} x_{p} E_{p i}(u) \in \mathcal{P}_{n} \otimes U\left(\mathfrak{g l}_{n}\right) \subset \Omega_{n} \otimes U\left(\mathfrak{g l}_{n}\right), \\
& \omega_{j}(v)=\sum_{q=1}^{n} e_{q} E_{q j}(v) \in \Lambda_{n} \otimes U\left(\mathfrak{g l}_{n}\right) \subset \Omega_{n} \otimes U\left(\mathfrak{g l}_{n}\right) .
\end{aligned}
$$

Their commutation relations are given as follows:

Lemma 3.1. For any parameters $u, v$ and $z$, we have

$$
\begin{aligned}
& \eta_{i}(u-1) \eta_{j}(u)-\eta_{j}(u-1) \eta_{i}(u)=0, \\
& \omega_{i}(v) \omega_{j}(v-1)+\omega_{j}(v) \omega_{i}(v-1)=0, \\
& \omega_{j}(u) \eta_{i}(v)-\eta_{i}(u) \omega_{j}(v)=x_{j} \omega_{i}(z)-\eta_{j}(z) e_{i} .
\end{aligned}
$$

Proof. These three assertions can be proved by direct computations, which are similar to each other. Since the proofs for (1) and (2) are found, e.g., in [U1-U4] and $[\mathrm{IU}]$, we give only the proof of (3) here. Compute the commutator:

$$
\begin{aligned}
{\left[\omega_{j}(v), \eta_{i}(u)\right]=\left[\omega_{j}, \eta_{i}\right] } & =\sum_{p, q} x_{p} e_{q}\left[E_{q j}, E_{p i}\right] \\
& =\sum_{p, q} x_{p} e_{q}\left(\delta_{j p} E_{q i}-\delta_{i q} E_{p j}\right) \\
& =\sum_{q} x_{j} e_{q} E_{q i}-\sum_{p} x_{p} e_{i} E_{p j} \\
& =x_{j} \omega_{i}-\eta_{j} e_{i}=x_{j} \omega_{i}(z)-\eta_{j}(z) e_{i} .
\end{aligned}
$$


This proves (3) as desired.

Let $I=\left(i_{1}, i_{2}, \cdots, i_{r}\right)$ be an $r$-tuple of non-negative integers with $1 \leq i_{k} \leq n$. We write $r=|I|$. The symmetric group $\mathfrak{S}_{r}$ of $r$ letters naturally acts on the set of such $r$-tuples by

$$
\left(i_{1}, i_{2}, \cdots, i_{r}\right)^{\sigma}=\left(i_{\sigma(1)}, i_{\sigma(2)}, \cdots, i_{\sigma(r)}\right) .
$$

We write $x^{I}=x_{i_{1}} x_{i_{2}} \cdots x_{i_{r}}$ and $e^{I}=e_{i_{1}} e_{i_{2}} \cdots e_{i_{r}}$. It is clear that

$$
x^{I^{\sigma}}=x^{I}, \quad e^{I^{\sigma}}=\operatorname{sgn}(\sigma) e^{I} .
$$

Furthermore we define for $r=|I|$,

$$
\begin{aligned}
\eta^{(I)}(u) & =\eta_{i_{1}}(u-r+1) \eta_{i_{2}}(u-r+2) \cdots \eta_{i_{r}}(u), \\
\omega^{(I)}(v) & =\omega_{i_{1}}(v) \omega_{i_{2}}(v-1) \cdots \omega_{i_{r}}(v-r+1) .
\end{aligned}
$$

From the commutation relations (1) and (2) in Lemma 3.1, we see

$$
\eta^{\left(I^{\sigma}\right)}(u)=\eta^{(I)}(u), \quad \omega^{\left(I^{\sigma}\right)}(v)=\operatorname{sgn}(\sigma) \omega^{(I)}(v) .
$$

These relations assure that the two assignments given by

$$
\boldsymbol{E}(u)^{\mathcal{P}}: x^{I} \mapsto \eta^{(I)}(u), \quad \boldsymbol{E}(v)^{\Lambda}: e^{I} \mapsto \omega^{(I)}(v)
$$

are well-defined, so that we obtain the linear maps

$$
\boldsymbol{E}(u)^{\mathcal{P}}: \mathcal{P}_{n} \longrightarrow \mathcal{P}_{n} \otimes U\left(\mathfrak{g l}_{n}\right), \quad \boldsymbol{E}(v)^{\Lambda}: \Lambda_{n} \longrightarrow \Lambda_{n} \otimes U\left(\mathfrak{g l}_{n}\right)
$$

The restrictions of these maps to the homogeneous pieces will be simply denoted by $\boldsymbol{E}(u)^{\mathcal{P}^{k}}=\left.\boldsymbol{E}(u)^{\mathcal{P}}\right|_{\mathcal{P}^{k}}$ and $\boldsymbol{E}(u)^{\Lambda^{k}}=\left.\boldsymbol{E}(u)^{\Lambda}\right|_{\Lambda^{k}}$.

From the definitions of these $\boldsymbol{E}(u)^{\mathcal{P}}$ and $\boldsymbol{E}(u)^{\Lambda}$ and the products $\times$ and $\check{x}$ in $\S 1$, we see the following product formulas according to the decompositions $\mathcal{P}^{k+h}=$ $\mathcal{P}^{k} \otimes \mathcal{P}^{h}$ and $\Lambda^{h+l}=\Lambda^{h} \otimes \Lambda^{l}$ :

$$
\begin{aligned}
& \boldsymbol{E}(u)^{\mathcal{P}^{k+h}}=\boldsymbol{E}(u-h)^{\mathcal{P}^{k}} \times \boldsymbol{E}(u)^{\mathcal{P}^{h}}, \\
& \boldsymbol{E}(u)^{\mathcal{P}^{k+h}}=\boldsymbol{E}(u-k)^{\mathcal{P}^{h}} \check{\times} \boldsymbol{E}(u)^{\mathcal{P}^{k}}, \\
& \boldsymbol{E}(v)^{\Lambda^{h+l}}=\boldsymbol{E}(v)^{\Lambda^{h} \times \boldsymbol{E}(v-h)^{\Lambda^{l}},} \\
& \boldsymbol{E}(v)^{\Lambda^{h+l}}=\boldsymbol{E}(v)^{\Lambda^{l}} \check{\times} \boldsymbol{E}(v-l)^{\Lambda^{h}} .
\end{aligned}
$$

Combining these two linear maps $\boldsymbol{E}(u)^{\mathcal{P}}$ and $\boldsymbol{E}(u)^{\Lambda}$, we can construct the linear maps of the following type:

$$
\begin{aligned}
& \boldsymbol{E}(u)^{\mathcal{P}} \times \boldsymbol{E}(v)^{\Lambda}: \Omega_{n} \longrightarrow \Omega_{n} \otimes U\left(\mathfrak{g l}_{n}\right), \\
& \boldsymbol{E}(v)^{\Lambda} \check{\times} \boldsymbol{E}(u)^{\mathcal{P}}: \Omega_{n} \longrightarrow \Omega_{n} \otimes U\left(\mathfrak{g l}_{n}\right) .
\end{aligned}
$$


From the associativity (1.3) and (1.4) of the products and the formulas (3.4), (3.5), $\left(3.4^{\vee}\right),\left(3.5^{\vee}\right)$ above, we also have the following product formulas.

$$
\begin{aligned}
& \boldsymbol{E}(u)^{\mathcal{P}^{k+h}} \times \boldsymbol{E}(v)^{\Lambda^{l}}=\boldsymbol{E}(u-h)^{\mathcal{P}^{k}} \times\left(\boldsymbol{E}(u)^{\mathcal{P}^{h}} \times \boldsymbol{E}(v)^{\Lambda^{l}}\right), \\
& \boldsymbol{E}(u)^{\mathcal{P}^{k}} \times \boldsymbol{E}(v)^{\Lambda^{h+l}}=\left(\boldsymbol{E}(u)^{\mathcal{P}^{k}} \times \boldsymbol{E}(v)^{\Lambda^{h}}\right) \times \boldsymbol{E}(v-h)^{\Lambda^{l}}, \\
& \boldsymbol{E}(v)^{\Lambda^{l}} \check{\times} \boldsymbol{E}(u)^{\mathcal{P}^{k+h}}=\left(\boldsymbol{E}(v)^{\Lambda^{l}} \check{\times} \boldsymbol{E}(u-k)^{\mathcal{P}^{h}}\right) \check{\times} \boldsymbol{E}(u)^{\mathcal{P}^{k}}, \\
& \boldsymbol{E}(v)^{\Lambda^{h+l}} \check{\times} \boldsymbol{E}(u)^{\mathcal{P}^{k}}=\boldsymbol{E}(v)^{\Lambda^{l}} \check{\times}\left(\boldsymbol{E}(v-l)^{\Lambda^{h}} \check{\times} \boldsymbol{E}(u)^{\mathcal{P}^{k}}\right) .
\end{aligned}
$$

We remark that the maps of this type with arbitrary parameters do not commute with the differentials $d, d^{*}$ in general. However, if we chose the parameters $u, v$ correctly, the maps $\boldsymbol{E}(u)^{\mathcal{P}} \times \boldsymbol{E}(v)^{\Lambda}$ do commute with the differentials. We will establish this kind of commutativity in Theorem 3.3 and Theorem $3.3^{*}$ after several computations. We point out here that our goal is somewhat delicate. As stated in $\S 2$, the differentials $d, d^{*}$ for the complex whose coefficients are extended by $U\left(\mathfrak{g l}_{n}\right)$, are to act trivially on $U\left(\mathfrak{g l}_{n}\right)$. By definition we have $d \eta_{i}(u)=\omega_{i}(u)$ and $d^{*} \omega_{j}(v)=\eta_{j}(v)$. From these and the derivation rule (2.2), we see that, for example, $d \eta^{(I)}(u)$ is computed as the sum of the terms

$\eta_{i_{1}}(u-r+1) \cdots \eta_{i_{p-1}}(u-r+p-1) \omega_{i_{p}}(u-r+p) \eta_{i_{p+1}}(u-r+p+1) \cdots \eta_{i_{r}}(u)$ for $1 \leq p \leq r=|I|$. For the proof the commutativity of the differential $d$ with the map of the form $\boldsymbol{E}(u)^{\mathcal{P}} \times \boldsymbol{E}(v)^{\Lambda}$ thus needs manipulation of the commutation relations among the factors, which are by no means obvious.

To make our computations clearer, we introduce some notation. For the $r$-tuple $I=\left(i_{1}, i_{2}, \cdots, i_{r}\right)$, we denote by

$$
I^{(p)}=\left(i_{1}, \cdots, i_{p-1}, i_{p+1}, \cdots, i_{r}\right)
$$

the $(r-1)$-tuple with the $p$-th component dropped from $I$. Using this, we see

$$
d x^{I}=\sum_{p=1}^{|I|} x^{I^{(p)}} e_{i_{p}}, \quad d^{*} e^{I}=\sum_{p=1}^{|I|}(-)^{p-1} x_{i_{p}} e^{I^{(p)}} .
$$

Furthermore as a computational tool, we introduce the difference operator $\Delta$ defined by $\Delta \varphi(u)=\varphi(u+1)-\varphi(u)$. Then we have

$$
\begin{aligned}
& \Delta(\varphi(u) \psi(u))=\Delta \varphi(u) \cdot \psi(u)+\varphi(u+1) \cdot \Delta \psi(u), \\
& \Delta(\varphi(u) \psi(u))=\Delta \varphi(u) \cdot \psi(u+1)+\varphi(u) \cdot \Delta \psi(u) .
\end{aligned}
$$

Note that these are valid even when $\varphi(u)$ and $\psi(u)$ do not necessarily commute. We apply these formulas repeatedly to the products (3.1) and (3.2). Then we see

$$
\begin{aligned}
\Delta \eta^{(I)}(u) & =\sum_{p=1}^{|I|} x_{i_{p}} \eta^{\left(I^{(p)}\right)}(u) \\
\Delta \omega^{(I)}(v) & =\sum_{p=1}^{|I|}(-)^{p-1} e_{i_{p}} \omega^{\left(I^{(p)}\right)}(v)
\end{aligned}
$$


The following computations are crucial in this paper.

Lemma 3.2. We have

$$
d \eta^{(I)}(u)=\left(\boldsymbol{E}(u)^{\mathcal{P}} \times \boldsymbol{E}(u-|I|+1)^{\Lambda}\right)\left(d x^{I}\right),
$$

and also

$$
d^{*} \omega^{(I)}(v)=\left(\boldsymbol{E}(v-|I|+1)^{\mathcal{P}} \times \boldsymbol{E}(v)^{\Lambda}\right)\left(d^{*} e^{I}\right) .
$$

Proof for (1). We prove (1) by induction on $r=|I|$. When $r=1$, the assertion is clear. Assume that we have proved the formula for the $r$-tuples $I=\left(i_{1}, i_{2}, \cdots, i_{r}\right)$, and we put $J=\left(i_{1}, i_{2}, \cdots, i_{r}, i_{r+1}\right)$. In the calculation below, we sometimes abbreviate $i=i_{r+1}$. Then we see $\eta^{(J)}(u)=\eta^{(I)}(u-1) \eta_{i}(u)$. By the derivation rule (2.2), we have

$$
d \eta^{(J)}(u)=d \eta^{(I)}(u-1) \eta_{i}(u)+\eta^{(I)}(u-1) \omega_{i}(u) .
$$

From (3.10) and the definition, our induction assumption turns to be

$$
d \eta^{(I)}(u)=\sum_{1 \leq p \leq r} \eta^{\left(I^{(p)}\right)}(u) \omega_{i_{p}}(u-r+1) .
$$

Then the first term in $d \eta^{J}(u)$ can be computed by Lemma 3.1 (3) with $z=u$ as:

$$
\begin{aligned}
d \eta^{(I)}(u-1) \eta_{i}(u)= & \sum_{1 \leq p \leq r} \eta^{\left(I^{(p)}\right)}(u-1) \omega_{i_{p}}(u-r) \eta_{i}(u) \\
= & \sum_{1 \leq p \leq r} \eta^{\left(I^{(p)}\right)}(u-1) \eta_{i}(u) \omega_{i_{p}}(u-r) \\
& +\sum_{1 \leq p \leq r} \eta^{\left(I^{(p)}\right)}(u-1)\left(x_{i_{p}} \omega_{i}(u)-\eta_{i_{p}}(u) e_{i}\right) \\
= & \sum_{1 \leq p \leq r} \eta^{\left(J^{(p)}\right)}(u) \omega_{i_{p}}(u-r) \\
& +\sum_{1 \leq p \leq r} x_{i_{p}} \eta^{\left(I^{(p)}\right)}(u-1) \omega_{i}(u)-\sum_{1 \leq p \leq r} \eta^{(I)}(u) e_{i} \\
= & \sum_{1 \leq p \leq r} \eta^{\left(J^{(p)}\right)}(u) \omega_{i_{p}}(u-r)+\Delta \eta^{(I)}(u-1) \omega_{i}(u)-r \eta^{(I)}(u) e_{i} .
\end{aligned}
$$

Here for the middle term in the last line, we have used (3.13). For the second term in $d \eta_{J}(u)$, we have an easy relation

$$
\eta^{(I)}(u) \omega_{i}(u-r)-\eta^{(I)}(u-1) \omega_{i}(u)=\Delta \eta^{(I)}(u-1) \cdot \omega_{i}(u)-r \eta^{(I)}(u) e_{i} .
$$


Then plugging this into the first term, we see

$$
\begin{aligned}
d \eta^{(J)}(u) & =\sum_{1 \leq p \leq r} \eta^{\left(J^{(p)}\right)}(u) \omega_{i_{p}}(u-r)+\eta^{(I)}(u) \omega_{i}(u-r) \\
& =\sum_{1 \leq p \leq r+1} \eta^{\left(J^{(p)}\right)}(u) \omega_{i_{p}}(u-r) .
\end{aligned}
$$

This concludes the induction.

Proof for (2). As above we prove (2) by induction on $r=|I|$ and the proof is parallel to $(1)$. Let $J=\left(i_{0}, i_{1}, \cdots, i_{r}\right)$ and $I=\left(i_{1}, \cdots, i_{r}\right)$. We sometimes write $i_{0}=i$ for simplicity. The induction assumption for $r$ can be given in the form

$$
d^{*} \omega^{(I)}(v)=\sum_{p=1}^{r}(-)^{p-1} \eta_{i_{p}}(v-r+1) \omega^{\left(I^{(p)}\right)}(v) .
$$

Since $\omega^{(J)}(v)=\omega_{i}(v) \omega^{(I)}(v-1)$, we have

$$
d^{*} \omega^{(J)}(v)=\eta_{i}(v) \omega^{(I)}(v-1)-\omega_{i}(v) d^{*} \omega^{(I)}(v-1) .
$$

From the induction assumption, the second term can be computed by (3) of Lemma 3.1 (3) with $z=v$ :

$$
\begin{aligned}
\omega_{i}(v) d^{*} \omega^{(I)}(v)= & \sum_{1 \leq p \leq r}(-)^{p-1} \omega_{i}(v) \eta_{i_{p}}(v-r) \omega^{\left(I^{(p)}\right)}(v-1) \\
= & \sum_{1 \leq p \leq r}(-)^{p-1} \eta_{i_{p}}(v-r) \omega_{i}(v) \omega^{\left(I^{(p)}\right)}(v-1) \\
& +\sum_{1 \leq p \leq r}(-)^{p-1}\left(x_{i} \omega_{i_{p}}(v)-\eta_{i}(v) e_{i_{p}}\right) \omega^{\left(I^{(p)}\right)}(v-1) \\
= & \sum_{1 \leq p \leq r}(-)^{p-1} \eta_{i_{p}}(v-r) \omega^{\left(J^{(p)}\right)}(v) \\
& +\sum_{1 \leq p \leq r} x_{i} \omega^{(I)}(v)-\sum_{1 \leq p \leq r}(-)^{p-1} \eta_{i}(v) e_{i_{p}} \omega^{\left(I^{(p)}\right)}(v-1) \\
= & \sum_{1 \leq p \leq r}(-)^{p-1} \eta_{i_{p}}(v-r) \omega^{\left(J^{(p)}\right)}(v) \\
& +r x_{i} \omega^{(I)}(v)-\eta_{i}(v) \Delta \omega^{(I)}(v-1) .
\end{aligned}
$$

Here for the last term in the last line, we used (3.14). For the first term in $d^{*} \omega_{J}(v)$, note the relation

$$
\eta_{i}(v) \omega^{(I)}(v-1)-\eta_{i}(v-r) \omega^{(I)}(v)=r x_{i} \omega^{(I)}(v)-\eta_{i}(v) \Delta \omega^{(I)}(v-1) .
$$


Plugging this into the above, we see

$$
\begin{aligned}
d^{*} \omega^{(J)}(u) & =\eta_{i}(v-r) \omega^{(I)}(v)-\sum_{1 \leq p \leq r}(-)^{p-1} \eta_{i_{p}}(v-r) \omega^{\left(J^{(p)}\right)}(v) \\
& =\sum_{0 \leq p \leq r}(-)^{p} \eta_{i_{p}}(v-r) \omega^{\left(J^{(p)}\right)}(v) .
\end{aligned}
$$

This completes the induction as desired.

Lemma 3.2 ${ }^{\vee}$. We have

$$
d \eta^{(I)}(u)=\left(\boldsymbol{E}(u)^{\Lambda} \check{\times} \boldsymbol{E}(u-1)^{\mathcal{P}}\right)\left(d x^{I}\right),
$$

and also

$$
d^{*} \omega^{(I)}(v)=\left(\boldsymbol{E}(v-1)^{\Lambda} \check{\times} \boldsymbol{E}(v)^{\mathcal{P}}\right)\left(d^{*} e^{I}\right)
$$

Since the proofs for this lemma are quite parallel to those for Lemma 3.2, we omit the proofs.

Theorem 3.3. The linear maps

$$
\boldsymbol{E}(u)^{\mathcal{P}^{k}} \times \boldsymbol{E}(u-k)^{\Lambda^{l}}: \Omega_{n}^{k, l} \longrightarrow \Omega_{n}^{k, l} \otimes U\left(\mathfrak{g l}_{n}\right)
$$

and

$$
\boldsymbol{E}(v)^{\Lambda^{l}} \check{\times} \boldsymbol{E}(v-l)^{\mathcal{P}^{k}}: \Omega_{n}^{k, l} \longrightarrow \Omega_{n}^{k, l} \otimes U\left(\mathfrak{g l}_{n}\right)
$$

commute with the differential d of the Koszul complex.

Proof. What we need to prove are

$$
d\left(\boldsymbol{E}(u)^{\mathcal{P}^{k}} \times \boldsymbol{E}(u-k)^{\Lambda^{l}}\right)=\left(\boldsymbol{E}(u)^{\mathcal{P}^{k-1}} \times \boldsymbol{E}(u-k+1)^{\Lambda^{l+1}}\right) d
$$

and

$$
d\left(\boldsymbol{E}(v)^{\Lambda^{l}} \check{\times} \boldsymbol{E}(v-l)^{\mathcal{P}^{k}}\right)=\left(\boldsymbol{E}(v)^{\Lambda^{l+1}} \check{\times} \boldsymbol{E}(v-l-1)^{\mathcal{P}^{k-1}}\right) d .
$$

Lemma $3.2(1)$ and Lemma $3.2^{\vee}(1)$ show that $(1)$ and $(2)$ are true for $\Omega_{n}^{k, 0}$. To obtain the general formulas, we combine the associativity (3.7) and (3.9) with the formulas (4), (5) below.

Let $\varphi \in \Omega_{n} \otimes U\left(\mathfrak{g l}_{n}\right)$ and $\psi \in \Lambda_{n} \otimes U\left(\mathfrak{g l}_{n}\right)$. Then from the derivation rule $(2.2)$ and $d \psi=0$, we see

$$
d(\varphi \psi)=d \varphi \cdot \psi, \quad d(\psi \varphi)=\psi \cdot d \varphi .
$$


These assure that the operator $d$ commutes with the multiplications $\times \boldsymbol{E}(v)^{\Lambda}$ and $\boldsymbol{E}(v)^{\Lambda} \check{x}$. In fact, for any linear map $\Phi$ from $\Omega_{n}$ to $\Omega_{n} \otimes U\left(\mathfrak{g l}_{n}\right)$, we have

$$
\begin{aligned}
d\left(\Phi \times \boldsymbol{E}(v)^{\Lambda}\right) & =d \Phi \times \boldsymbol{E}(v)^{\Lambda}, & & \left(\Phi \times \boldsymbol{E}(v)^{\Lambda}\right) d=\Phi d \times \boldsymbol{E}(v)^{\Lambda} \\
d\left(\boldsymbol{E}(v)^{\Lambda} \check{\times} \Phi\right) & =\boldsymbol{E}(v)^{\Lambda} \check{\times} d \Phi, & & \left(\boldsymbol{E}(v)^{\Lambda} \check{\times} \Phi\right) d=\boldsymbol{E}(v)^{\Lambda} \check{\times} \Phi d .
\end{aligned}
$$

By (4), Lemma $3.2(1)$ and (3.7), we see for (1)

$$
\begin{aligned}
d\left(\boldsymbol{E}(u)^{\mathcal{P}^{k}} \times \boldsymbol{E}(u-k)^{\Lambda^{l}}\right) & =d \boldsymbol{E}(u)^{\mathcal{P}^{k}} \times \boldsymbol{E}(u-k)^{\Lambda^{l}} \\
& =\left(\boldsymbol{E}(u)^{\mathcal{P}^{k-1}} \times \boldsymbol{E}(u-k+1)^{\Lambda^{1}}\right) d \times \boldsymbol{E}(u-k)^{\Lambda^{l}} \\
& =\left(\left(\boldsymbol{E}(u)^{\mathcal{P}^{k-1}} \times \boldsymbol{E}(u-k+1)^{\Lambda^{1}}\right) \times \boldsymbol{E}(u-k)^{\Lambda^{l}}\right) d \\
& =\left(\boldsymbol{E}(u)^{\mathcal{P}^{k-1}} \times \boldsymbol{E}(u-k+1)^{\Lambda^{l+1}}\right) d,
\end{aligned}
$$

and for (2) by (5), Lemma $3.2(2)$ and (3.9)

$$
\begin{aligned}
d\left(\boldsymbol{E}(v)^{\Lambda^{l}} \check{\times} \boldsymbol{E}(v-l)^{\mathcal{P}^{k}}\right) & =\boldsymbol{E}(v)^{\Lambda^{l}} \check{\times} d \boldsymbol{E}(v-l)^{\mathcal{P}^{k}} \\
& =\boldsymbol{E}(v)^{\Lambda^{l}} \check{\times}\left(\boldsymbol{E}(v-l)^{\Lambda^{1}} \check{\times} \boldsymbol{E}(v-l-1)^{\mathcal{P}^{k-1}}\right) d \\
& =\left(\boldsymbol{E}(v)^{\Lambda^{l}} \check{\times}\left(\boldsymbol{E}(v-l)^{\Lambda^{1}} \check{\times} \boldsymbol{E}(v-l-1)^{\mathcal{P}^{k-1}}\right)\right) d \\
& =\left(\boldsymbol{E}(v)^{\Lambda^{l+1}} \check{\times} \boldsymbol{E}(v-l-1)^{\mathcal{P}^{k-1}}\right) d .
\end{aligned}
$$

These prove the assertions (1) and (2).

The following theorem is parallel to Theorem 3.3.

Theorem 3.3*. The linear maps

$$
\boldsymbol{E}(v-l)^{\mathcal{P}^{k}} \times \boldsymbol{E}(v)^{\Lambda^{l}}: \Omega_{n}^{k, l} \longrightarrow \Omega_{n}^{k, l} \otimes U\left(\mathfrak{g l}_{n}\right)
$$

and

$$
\boldsymbol{E}(u-k)^{\Lambda^{l}} \check{\times} \boldsymbol{E}(u)^{\mathcal{P}^{k}}: \Omega_{n}^{k, l} \longrightarrow \Omega_{n}^{k, l} \otimes U\left(\mathfrak{g l}_{n}\right)
$$

commute with the differential $d^{*}$ of the Koszul complex.

Proof. The proofs for these assertions are similar to those for Theorem 3.3, so that we avoid the repetition. Use Lemma 3.2 (2), Lemma $3.2^{\vee}(2),(3.6)$ and (3.8) here, in place of Lemma $3.2(1)$, Lemma $3.2^{\vee}(1),(3.7)$ and (3.9) for Theorem 3.3. 


\section{Relations between certain central elements in $U\left(\mathfrak{g l}_{n}\right)$}

Theorems 3.3 and $3.3^{*}$ combined with Lemma 1.3 and Lemma 2 show the following:

Theorem 4.1. For $N \geq 1$, we have

$$
\begin{aligned}
& \sum_{k=0}^{N}(-)^{k} \operatorname{Tr}\left(\boldsymbol{E}(u)^{\mathcal{P}^{k}}\right) \operatorname{Tr}\left(\boldsymbol{E}(u-k)^{\Lambda^{N-k}}\right)=0 \\
& \sum_{l=0}^{N}(-)^{l} \operatorname{Tr}\left(\boldsymbol{E}(v)^{\Lambda^{l}}\right) \operatorname{Tr}\left(\boldsymbol{E}(v-l)^{\mathcal{P}^{N-l}}\right)=0
\end{aligned}
$$

and

$$
\begin{aligned}
& \sum_{l=0}^{N}(-)^{l} \operatorname{Tr}\left(\boldsymbol{E}(v-l)^{\mathcal{P}^{N-l}}\right) \operatorname{Tr}\left(\boldsymbol{E}(v)^{\Lambda^{l}}\right)=0, \\
& \sum_{k=0}^{N}(-)^{k} \operatorname{Tr}\left(\boldsymbol{E}(u-k)^{\Lambda^{N-k}}\right) \operatorname{Tr}\left(\boldsymbol{E}(u)^{\mathcal{P}^{k}}\right)=0 .
\end{aligned}
$$

Note that the difference between the relations (1) and (4) (or respectively (2) and (3)) is only in the order of multiplication of $\operatorname{Tr}\left(\boldsymbol{E}(u)^{\mathcal{P}^{k}}\right)$ and $\operatorname{Tr}\left(\boldsymbol{E}(v)^{\Lambda^{l}}\right)$. Actually since these traces $\operatorname{Tr}\left(\boldsymbol{E}(u)^{\mathcal{P}^{k}}\right)$ and $\operatorname{Tr}\left(\boldsymbol{E}(v)^{\Lambda^{l}}\right)$ are seen to be central in $U\left(\mathfrak{g l}_{n}\right)$, the equalities (1) and (4) (or respectively (2) and (3)) express substantially the same identity.

To take a closer look at these traces, recall the action of the symmetric group $\mathfrak{S}_{r}$ on the set of $r$-tuples as described in $\S 3$. In the $\mathfrak{S}_{r}$-orbit $I^{\mathfrak{S}_{r}}$ of an $r$-tuple $I$, there is a unique $r$-tuple $I^{b}=\left(j_{1}, j_{2}, \cdots, j_{r}\right)$ satisfying $1 \leq j_{1} \leq j_{2} \leq \cdots \leq j_{r} \leq n$. Let $\alpha_{p}$ be the multiplicity of the letter $p$ in $I$. Then the $I^{b}$ takes the form

$$
I^{b}=(\overbrace{1, \cdots, 1}^{\alpha_{1}}, \overbrace{2, \cdots, 2}^{\alpha_{2}}, \cdots, \overbrace{n, \cdots, n}^{\alpha_{n}}) .
$$

In this way, the representative $I^{b}$ of the $\mathfrak{S}_{r}$-orbit is identified with the multi-index $\alpha=\left(\alpha_{1}, \alpha_{2}, \cdots, \alpha_{n}\right)$ consisting of non-negative integers. For a multi-index $\alpha$, we denote by $I_{\alpha}$ the corresponding $r$-tuple with non-decreasing entries, and by $i_{\alpha}$ the $i$-th component of $I_{\alpha}$, i.e., $I_{\alpha}=\left(1_{\alpha}, 2_{\alpha}, \cdots, r_{\alpha}\right)$. As usual we put $|\alpha|=$ $\alpha_{1}+\alpha_{2}+\cdots+\alpha_{n}=r$ and $\alpha !=\alpha_{1} ! \alpha_{2} ! \cdots \alpha_{n}$ !. Identifying the multi-index $\alpha$ with $I_{\alpha}$, we use the notation $x^{\alpha}=x^{I_{\alpha}}=x_{1}^{\alpha_{1}} x_{2}^{\alpha_{2}} \cdots x_{n}^{\alpha_{n}}$ and $e^{\alpha}=e^{I_{\alpha}}=e_{1}^{\alpha_{1}} e_{2}^{\alpha_{2}} \cdots e_{n}^{\alpha_{n}}$. For the latter $e^{\alpha}=0$ if some of $\alpha_{p}$ is greater than 1. It is clear that the set $\left\{x^{\alpha} ;|\alpha|=k\right\}$ forms a basis of $\mathcal{P}_{n}^{k}$ and that the set $\left\{e^{\alpha} ;|\alpha|=l, \alpha_{p} \in\{0,1\}\right\}$ forms a basis of $\Lambda_{n}^{l}$. 
With this notation, we can describe how to use multi-indices to form a matrix $A^{\alpha \beta}$ from a given $n \times n$ matrix. More specifically, for an $n \times n$ matrix $A$ and for multi-indices $\alpha, \beta$, we denote by $A^{\alpha \beta}$ the $|\alpha| \times|\beta|$ matrix whose $(i, j)$ component is given by $A_{i j}^{\alpha \beta}=A_{i_{\alpha} j_{\beta}}$. Note that if all the components of $\alpha, \beta$ consist of either 0 or 1 , this $A^{\alpha \beta}$ is nothing but the submatrix whose rows and columns are respectively determined by $I_{\alpha}$ and $I_{\beta}$.

We use this convention for the matrices

$$
\boldsymbol{E}=\left(E_{i j}\right)_{i, j=1}^{n}, \quad \mathbf{1}=\left(\delta_{i j}\right)_{i, j=1}^{n}
$$

to define

$$
\boldsymbol{E}_{\mathcal{P} \sharp}^{\alpha \beta}(u)=\boldsymbol{E}^{\alpha \beta}+\mathbf{1}^{\alpha \beta} \cdot(u-\operatorname{diag}(r-1, r-2, \cdots, 1,0)),
$$

and

$$
\boldsymbol{E}_{\Lambda \downarrow}^{\alpha \beta}(u)=\boldsymbol{E}^{\alpha \beta}-\mathbf{1}^{\alpha \beta} \cdot(u-\operatorname{diag}(r-1, r-2, \cdots, 1,0)),
$$

for multi-indices $\alpha, \beta$ with $r=|\alpha|=|\beta|$.

For an $r \times r$ matrix $A=\left(A_{i j}\right)_{i, j=1}^{r}$ whose entries are from an associative algebra $\mathcal{A}$, we define in general its permanent and determinant by

$$
\begin{aligned}
\operatorname{per}(A) & =\sum_{\sigma \in \mathfrak{S}_{r}} A_{\sigma(1) 1} A_{\sigma(2) 2} \cdots A_{\sigma(r) r} \\
\operatorname{det}(A) & =\sum_{\sigma \in \mathfrak{S}_{r}} \operatorname{sgn}(\sigma) A_{\sigma(1) 1} A_{\sigma(2) 2} \cdots A_{\sigma(r) r}
\end{aligned}
$$

The matrix element of the linear maps $\boldsymbol{E}(u)^{\mathcal{P}^{k}}$ and $\boldsymbol{E}(u)^{\Lambda^{k}}$ are expressed by the permanent and the determinant as follows. They are verified by direct computations.

Lemma 4.2. For $|\beta|=k$, we have the following

$$
\begin{aligned}
& \boldsymbol{E}(u)^{\mathcal{P}}\left(x^{\beta}\right)=\sum_{|\alpha|=k} x^{\alpha} \frac{\operatorname{per}\left(\boldsymbol{E}_{\mathcal{P} \sharp}^{\alpha \beta}(u)\right)}{\alpha !}, \\
& \boldsymbol{E}(v)^{\Lambda}\left(e^{\beta}\right)=\sum_{|\alpha|=k} e^{\alpha} \operatorname{det}\left(\boldsymbol{E}_{\Lambda \downarrow}^{\alpha \beta}(k-1-v)\right) .
\end{aligned}
$$

As the traces of these linear maps, we define

$$
\begin{aligned}
& D_{k}(u)=\operatorname{Tr}\left(\boldsymbol{E}(u)^{\mathcal{P}^{k}}\right)=\sum_{|\alpha|=k} \frac{1}{\alpha !} \operatorname{per}\left(\boldsymbol{E}_{\mathcal{P} \sharp}^{\alpha \alpha}(u)\right), \\
& C_{k}(v)=\operatorname{Tr}\left(\boldsymbol{E}(k-1-v)^{\Lambda^{k}}\right)=\sum_{|\alpha|=k} \operatorname{det}\left(\boldsymbol{E}_{\Lambda \natural}^{\alpha \alpha}(v)\right),
\end{aligned}
$$


and $D_{k}=D_{k}(0), C_{k}=C_{k}(0)$. Note that the determinant $\operatorname{det}\left(\boldsymbol{E}_{\Lambda \boxminus}^{\alpha \alpha}(v)\right)$ vanishes if $\alpha_{p}>1$ for some $p$, so that we may put the restriction $\alpha_{p}=0,1$ on $\alpha$ in the summation above for $C_{k}(v)$. We also remark that the expressions of $D_{k}(u)$ and $C_{k}(v)$ introduced above as the traces of the maps $\boldsymbol{E}(u)^{\mathcal{P}^{k}}$ and $\boldsymbol{E}(u)^{\Lambda^{k}}$ give the basis of the fact that they are central in $U\left(\mathfrak{g l}_{n}\right)$ (cf. [Na1], [Ok], [U3], [U4]). It is easy to see that these $D_{k}(u)$ and $C_{k}(u)$ satisfy the difference relations $\Delta D_{k}(u)=$ $(n+k-1) D_{k-1}(u)$ and $\Delta C_{k}(u)=(k-n-1) C_{k-1}(u)$, so that they are respectively expressed by polynomials in $u$ with coefficients $D_{k}$ and $C_{k}$ :

$$
\begin{aligned}
& D_{k}(u)=\sum_{r=0}^{k} u^{(r)}\left(\begin{array}{c}
n+k-1 \\
r
\end{array}\right) D_{k-r}, \\
& C_{k}(u)=\sum_{r=0}^{k} u^{(r)}\left(\begin{array}{c}
k-n-1 \\
r
\end{array}\right) C_{k-r},
\end{aligned}
$$

where $u^{(r)}=u(u-1) \cdots(u-r+1)$. For the proofs of these expansions, see [U4, (2.9)] for the $D_{k}$ case, and [U3, Prop. 4] for the $C_{k}$ case. Note also that by definition $C_{k}(v)=0$ for $k>n$ and the expression above for $C_{k}(u)$ is compatible with this, because $C_{k}=0$ for $k>n$. From Theorem 4.1 above, we see

Theorem 4.3 (Wronski Relations). For $N \geq 1$, we have

$$
\begin{aligned}
& \sum_{k=0}^{N}(-)^{k} D_{k}(u) C_{N-k}(N-1-u)=0 \\
& \sum_{l=0}^{N}(-)^{l} C_{l}(l-1-v) D_{N-l}(v-l)=0 .
\end{aligned}
$$

One can observe asymmetry in the formulas (1) and (2) in Theorem 4.3. However, these formulas are converted into the following relations between the two (infinite) matrices

$$
\boldsymbol{D}(u) \boldsymbol{C}(u)=1, \quad \boldsymbol{C}(u) \boldsymbol{D}(u)=1,
$$

where $\boldsymbol{C}(u)=\left(\boldsymbol{C}(u)_{i j}\right)_{i, j=0}^{\infty}$ and $\boldsymbol{D}(u)=\left(\boldsymbol{D}(u)_{i j}\right)_{i, j=0}^{\infty}$ are the matrices with the entries

$$
\boldsymbol{C}(u)_{i j}=(-)^{j-i} C_{j-i}(j-1-u), \quad \boldsymbol{D}(u)_{i j}=D_{j-i}(u-i)
$$

under the conventions $C_{k}(u)=0$ and $D_{k}(u)=0$ for $k<0$. Thus the symmetry and the equivalence between the two formulas in Theorem 4.3 is now clear. (Note that, since the matrices $\boldsymbol{C}(u)$ and $\boldsymbol{D}(u)$ are upper triangular (although of the infinite size), either one of the equations (4.1) does imply the other.) Furthermore, from (4.1), computing a suitable entry giving $D_{k}(u)$ and $C_{l}(v)$ by Cramer's rule, we 
have the following determinant expressions (cf. [He, p. 17], [KW]). Note that the formulas in Theorem 4.4 are totally symmetric in $D_{k}(u)$ and $C_{l}(v)$.

Theorem 4.4 (Wronski Formulas). An explicit determinant expression of $D_{k}(u)$ by $C_{k}(u)$ is given by the following formula:

$D_{k}(u)=\operatorname{det}\left[\begin{array}{ccccc}C_{1}(-u) & C_{2}(1-u) & \cdots & C_{k-1}(k-2-u) & C_{k}(k-1-u) \\ 1 & C_{1}(1-u) & \cdots & C_{k-2}(k-2-u) & C_{k-1}(k-1-u) \\ & 1 & \ddots & \vdots & \vdots \\ & & \ddots & C_{1}(k-2-u) & C_{2}(k-1-u) \\ 0 & & & 1 & C_{1}(k-1-u)\end{array}\right]$.

Also a determinant expression of $C_{l}(u)$ by $D_{l}(u)$ is given by

$$
C_{l}(l-1-v)=\operatorname{det}\left[\begin{array}{ccccc}
D_{1}(v) & D_{2}(v) & \cdots & D_{l-1}(v) & D_{l}(v) \\
1 & D_{1}(v-1) & \cdots & D_{l-2}(v-1) & D_{l-1}(v-1) \\
& 1 & \ddots & \vdots & \vdots \\
0 & & \ddots & D_{1}(v-l+2) & D_{2}(v-l+2) \\
& & & 1 & D_{1}(v-l+1)
\end{array}\right]
$$

or equivalently

$$
C_{l}(v)=\operatorname{det}\left[\begin{array}{ccccc}
D_{1}(l-1-v) & D_{2}(l-1-v) & \cdots & D_{l-1}(l-1-v) & D_{l}(l-1-v) \\
1 & D_{1}(l-2-v) & \cdots & D_{l-2}(l-2-v) & D_{l-1}(l-2-v) \\
& 1 & \ddots & \vdots & \vdots \\
0 & & \ddots & D_{1}(1-v) & D_{2}(1-v) \\
& & & 1 & D_{1}(-v)
\end{array}\right] .
$$

Remark. Via the natural interpretation of the central elements of $U\left(\mathfrak{g l}_{n}\right)$ in terms of symmetric functions, the elements $C_{k}(u)$ and $D_{k}(u)$ correspond respectively to the shifted Schur functions $e_{k}^{*}\left(x_{1}-u, \cdots, x_{n}-u\right)$ and $h_{k}^{*}\left(x_{1}+u, \cdots, x_{n}+u\right)$. Then the Wronski formula follows from Proposition 11.5 (Jacobi-Trudy formula) of $[\mathrm{OO}]$. The author would like to thank the referee for pointing out this fact together with the reference. 


\section{References}

[A] A. C. Aitken, Determinants and Matirces, Oliver and Boyed, 1939.

[B] N. Bourbaki, Algèbre homologique (Éléments de Mathématique: Algèbre Chapitre 10), Masson, 1980.

[Ca1] A. Capelli, Über die Zurückführung der Cayley'schen Operation $\Omega$ auf gewöhnliche PolarOperationen, Math. Ann. 29 (1887), 331-338.

[Ca2] A. Capelli, Sur les opérations dans la théorie des formes algébriques, Math. Ann. 37 (1890), 1-37.

[Ge] I. M. Gelfand, Center of the infinitesimal groups, Mat. Sb. Nov. Ser. 26 (68) (1950), 103-112; English transl. in "Collected Papers" Vol. II, 22-30.

[He] P. Henrici, Applied and Computational Complex Analysis, vol. 1, Wiley, 1974.

[H1] R. Howe, Remarks on classical invariant theory, Trans. Amer. Math. Soc. 313 (1989), 539-570; Erratum Trans. Amer. Math. Soc. 318 (1990), 823.

[H2] R. Howe, Dual pairs in physics: Harmonic oscillators, photons, electrons, and singletons, Lectures in Applied Math. 21 (1985), 179-207.

[HU] R. Howe and T. Umeda, The Capelli identity, the double commutant theorem, and multiplicity-free actions, Math. Ann. 290 (1991), 565-619.

[I1] M. Itoh, Explicit Newton's formula for $\mathfrak{g l}_{n}$, J. Alg. 208 (1998), 687-697.

[I2] M. Itoh, Capelli elements for the orthogonal Lie algebras, J. Lie Theory 10 (2000), 463489.

[I3] M. Itoh, A Cayley-Hamilton theorem for the skew Capelli elements, J. Alg. 242 (2001), $740-761$.

[IU] M. Itoh and T. Umeda, On the central elements in the universal enveloping algebras of the orthogonal Lie algebras, Compositio Math. 127 (2001), 333-359.

[KW] K. Kinoshita and M. Wakayama, Explicit Capelli identities for skew symmeteric matrices, Proc. Edinburgh Math. Soc. 45 (2002), 449-465.

[Kz] J.-L. Koszul, Les algèbre de Lie graduée de type $\mathfrak{s l}(n, 1)$ et l'opérateur de A. Capelli, C.R. Acad. Sc. Paris 292 (1981), 139-141.

[M1] A. Molev, Sklyanin determinant, Laplace operators, and characteristic identities for classical Lie algebras, J. Math. Phys. 36 (1995), 923-943.

[M2] A. Molev, Factorial supersymmetric Schur functions and super Capelli identities, in: Kirillov's Seminar on Representation Theory (ed. G. I. Olshanski), AMS Translations, Series 2181 (1998), 109-137.

[MN] A. Molev and M. Nazarov, Capelli identities for classical Lie algebras, Math. Ann. 313 (1999), 315-357.

[MNO] A. Molev, M. Nazarov and G. Olshanskii, Yangians and classical Lie algebras, Russian Math. Surveys 51 (1996), 205-282.

[Na1] M. Nazarov, Quantum Berezinian and the classical Capelli identity, Lett. Math. Phys. 21 (1991), 123-131.

[Na2] M. Nazarov, Yangians and Capelli identities, in: Kirillov's Seminar on Representation Theory (ed. G. I. Olshanski), AMS Translations, Series 2181 (1998), 139-163.

[NUW] M. Noumi, T. Umeda and M. Wakayama, A quantum analogue of the Capelli identity and an elementary differential calculus on $G L_{q}(n)$, Duke Math. J. 76 (1994), 567-594.

[Ok] A. Okounkov, Quantum immanants and higher Capelli identities, Transformation Groups 1 (1996), 99-126.

[OO] A. Okounkov and G. Olshanski, Shfited Schur functions, St. Petersburg Math. J. 9 (1998), 239-300.

[T1] H. W. Turnbull, Theory of Equations, Oliver and Boyed, 1939.

[T2] H. W. Turnbull, The Theory of Determinants, Matrices, and Invariants, Dover, 1960.

[T3] H. W. Turnbull, Symmetric determinants and the Cayley and Capelli operators, Proc. Edinburgh Math. Soc. Ser. 28 (1948), 76-86. 
[U1] T. Umeda, The Capelli identities, a century after, Sugaku 46 (1994), 206-227 (in Japanese). English transl. in "Selected Papers on Harmonic Analysis, Groups, and Invariants" (ed. by K. Nomizu), AMS Translations, Series 2, 183 (1998), 51-78,

[U2] T. Umeda, Newton's formula for $\mathfrak{g l}_{n}$, Proc. Amer. Math. Soc. 126 (1998), 3169-3175.

[U3] T. Umeda, On the proof of the Capelli identities, preprint, 1997.

[U4] T. Umeda, On Turnbull identity for skew symmetric matrices, Proc. Edinburgh Math. Soc. 43 (2000), 379-393.

[Wy] H. Weyl, The Classical Groups, their Invariants and Representations, Princeton Univ. Press. 1946.

[Z] D. P. Želobenko Compact Lie Groups and their Representations, Transl. Math. Monographs 40 Amer. Math. Soc., 1973.

Tôru Umeda

Department of Mathematics

Faculty of Science

Kyoto University

Kyoto 606-8502

Japan

(Received: February 29, 2000) 